\title{
Validation of the determination of amino acids in plasma by high-performance liquid chromatography using automated pre-column derivatization with $o$-phthaldialdehyde
}

\author{
Durk Fekkes*, Astrid van Dalen, Margriet Edelman, Ans Voskuilen \\ Section Pathophysiology of Behaviour, Erasmus University Medical School, P.O. Box 1738, 3000 DR Rotterdam. Netherlands
}

First received 21 December 1994; revised manuscript received 24 February 1995; accepted 24 February 1995

\begin{abstract}
A sensitive and reproducible fully automated method for the determination of amino acids in plasma based on reversed-phase high-performance liquid chromatography and $o$-phthaldialdehyde pre-column derivatization is described. A $5-\mu \mathrm{m}$ Spherisorb ODS 2 column $(125 \times 3 \mathrm{~mm}$ I.D. $)$ was selected for routine determination. Over 40 physiological amino acids could be determined within 49 min (injection to injection) and 48 samples could be processed unattended. The coefficients of variation for most amino acids in plasma were below $4 \%$. We were also able to measure trace amounts of amino acids in plasma normally not detected in a routine analysis. The results obtained with the method described compared favourably with those of conventional amino acid analysis $(r=0.997)$ and were in excellent agreement with those of other laboratories $(r=0.999)$.
\end{abstract}

\section{Introduction}

Up to now, several methods have been described for the determination of amino acids in human plasma using automated $o$-phthaldialdehyde (OPA) pre-column derivatization and high-performance liquid chromatography (HPLC) [1-13]. Emphasis has been given to the reduced time required for analysis $[5,12,13]$. This is however not the only criterion for a successful amino acid analysis. It is also important to measure as many plasma amino acids as possible occurring with the OPA derivatization method with a satisfactory resolution $[3,10]$. Another

\footnotetext{
* Corresponding author.
}

problem encountered in most analyses based on HPLC using the OPA method is the reliability of the measured amino acid concentrations in human plasma $[4,6,7,13]$. This not only depends on the choice of the analytical column or the derivatization method, but also on the sample preparation [7].

The purpose of the present study was to develop a suitable, sensitive and quick, fully automated analysis for the quantitative determination of amino acids in plasma and other biological materials, by using OPA pre-column derivatization and separation by gradient elution reversed-phase HPLC. Analytical columns from several manufacturers were tested for this purpose. 


\section{Experimental}

\subsection{Equipment}

The HPLC system consisted of an HP 1050 Series quaternary pump (Hewlett-Packard, Waldbronn, Germany), a Model GT-103 on-line degassing device (Lab Quatec, Tokyo, Japan) and a Mistral column oven with a built-in Peltier cooling device (Spark Holland, Emmen, The Netherlands). The latter device was necessary in order to keep the column temperature exactly at $25^{\circ} \mathrm{C}$. For the automated precolumn derivatization a Model 231-401 Auto-sampling injector (Gilson, Villiers-le-Bel, France), equipped with a Model 1200 coldblock (Grey Line Engineering, MD, USA), and a Rheodyne injection valve 7010 with a $20-\mu \mathrm{l}$ filling loop were used. Fluorescence was monitored with a Model LC-240 fluorescence detector (Perkin-Elmer, Beaconfield, UK) equipped with a xenon discharge lamp and a $7-\mu l$ flow cell. Measurements were made at an excitation wavelength of $337 \mathrm{~nm}$ and an emission wavelength of $452 \mathrm{~nm}$ with a fixed spectral bandpass of $10 \mathrm{~nm}$. Data were collected on-line and processed by a PC Integration Pack data acquisition system (Kontron Instruments, Milan, Italy).

The following columns were utilized: Spherisorb ODS 2, $5 \mu \mathrm{m}, 150 \times 4.6 \mathrm{~mm}$ I.D., $150 \times 2 \mathrm{~mm} \mathrm{I.D}$. and $150 \times 3 \mathrm{~mm}$ I.D. (Shandon Scientific, Astmoor, U.K.); Hypersil ODS, 5 $\mu \mathrm{m}, 200 \times 2.1 \mathrm{~mm}$ I.D. (Hewlett-Packard, Waldbronn, Germany), Inertsil ODS-2, $5 \mu \mathrm{m}, 150 \times$ $2.1 \mathrm{~mm} \mathrm{I.D.}$, and Inertsil ODS-80A, $5 \mu \mathrm{m}$, $150 \times 4.6 \mathrm{~mm}$ I.D. (GL Sciences, Tokyo, Japan); Zorbax SB-C18, $3.5 \mu \mathrm{m}, 150 \times 4.6 \mathrm{~mm}$ I.D., and $5 \mu \mathrm{m}, 250 \times 3 \mathrm{~mm}$ I.D., and Zorbax SB-C8, 5 $\mu \mathrm{m}, 250 \times 4.6 \mathrm{~mm}$ I.D. (Rockland Technologies, DE, USA); Hypersil Amino Acid, $3 \mu \mathrm{m}, 150 \times$ $4.6 \mathrm{~mm}$ I.D. (Shandon Scientific, Astmoor, UK); Supelcosil LC-18, $5 \mu \mathrm{m}, 150 \times 4 \mathrm{~mm}$ I.D. (Supelco, Bellefonte, PA, USA); Spherisorb ODS 2 cartridge column, $5 \mu \mathrm{m}$ and $3 \mu \mathrm{m}$, $125 \times 3 \mathrm{~mm}$ I.D. (Phase Separations, Queensferry, UK). All analytical columns were protected by a Hypersil ODS guard column, 5 $\mu \mathrm{m}, 20 \times 2.1 \mathrm{~mm}$ I.D. (Hewlett-Packard, Waldbronn, Germany).

\subsection{Reagents and solvents}

All solutions were prepared with ultra pure water, generated by a Model UHQ-MK II Elgastat water purification system (Elga, Lane End, UK). All chemicals used were of analytical grade and the solvents of chromatographic grade. The amino acids, including a physiological standard, and propionic acid were purchased from Sigma (St. Louis, MO, USA). 2-Mercaptoethanol (2ME) was obtained from Fluka (Buchs, Switzerland). All other chemicals and solvents were purchased from Merck (Darmstadt, Germany).

The derivatization reagent was prepared by dissolving $50 \mathrm{mg}$ of OPA in $1 \mathrm{ml}$ of methanol, adding $9 \mathrm{ml}$ of deoxygenated $(30 \mathrm{~min}$ bubbling with nitrogen) sodium borate buffer $(0.2 \mathrm{~mol} / \mathrm{l}$,

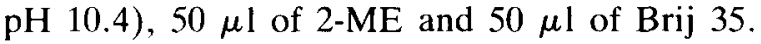
This reagent was kept in the dark at $4^{\circ} \mathrm{C}$ and was prepared freshly every two weeks. For analysis the OPA-reagent was diluted $(1: 1, \mathrm{v} / \mathrm{v})$ with borate buffer and placed in a $0.8 \mathrm{ml}$ amber crimp top vial with a silicone rubber, PTFE coated cap (Phase Separations). The OPA concentration in the reagent was $17 \mathrm{mmol} / \mathrm{l}$.

Solvents $\mathrm{A}$ and $\mathrm{B}$ both consisted of disodium hydrogen phosphate $(250 \mathrm{mmol} / \mathrm{l})$-propionic acid (250 $\mathrm{mmol} / \mathrm{l})$-acetonitrile-tetrahydrofuran-water $(20: 20: 7: 0.2: 51, v / v)$. The difference between the solvents was that prior to the addition of acetonitrile, tetrahydrofuran and water, the $\mathrm{pH}$ of solvent $\mathrm{A}$ was adjusted to 6.5 with $4 M \mathrm{NaOH}$ and the $\mathrm{pH}$ of solvent $\mathrm{B}$ was adjusted to 5.75 with concentrated propionic acid. The ultimate $\mathrm{pH}$ of solvents $\mathrm{A}$ and $\mathrm{B}$ ranged between $6.72-6.77$ and 5.95-6.00, respectively. The composition of solvent $\mathrm{C}$ was acetonitrile-methanol-dimethylsulfoxide-water $(28: 24: 5: 43, \mathrm{v} / \mathrm{v})$. All solvents were filtered through a $0.45-\mu \mathrm{m}$ filter by suction filtration.

Amino acid standards were prepared by mixing the physiological standard (Sigma) with a mixture of phosphoserine, asparagine, glutamine, phosphoethanolamine, hypotaurine, homoserine and the internal standard norvaline. The final concentration of most amino acids was $250 \mu \mathrm{mol} / \mathrm{l}$, except for phosphoserine, asparagine, phosphoethanolamine, $\mathrm{L}$-cystathionine and allo-L-cystathionine (125 $\mu \mathrm{mol} / \mathrm{l})$, hypo- 
taurine $(50 \mu \mathrm{mol} / \mathrm{l})$, tryptophan (TRP, 140-170 $\mu \mathrm{mol} / \mathrm{l})$, and homoserine and norvaline (400 $\mu \mathrm{mol} / \mathrm{l})$. The standard prepared in this way was diluted $(1: 1, \mathrm{v} / \mathrm{v})$ with solvent $\mathrm{A}$ in order to increase the stability of tryptophan and to match the peak heights with those in a plasma sample. Since the concentration of tryptophan in the physiological standard differed between batches, we determined its concentration by means of a range of tryptophan concentrations (20-400 $\mu \mathrm{mol} / \mathrm{l})$ prepared separately.

\subsection{Sample preparation}

Blood $(4 \mathrm{ml})$ was obtained from 44 healthy young volunteers (19 men and 25 women; age range $28-45$ years) and collected in siliconated vacutainer tubes containing $0.15 \% \mathrm{~K}_{3}$-EDTA as anticoagulant. Platelet poor plasma was obtained by centrifugation at $2650 \mathrm{~g}_{\max }$ for $20 \mathrm{~min}$ at $20^{\circ} \mathrm{C}$ in a Hettich Rotanta centrifuge and stored at $-30^{\circ} \mathrm{C}$ until analysis. In order to check proper centrifugation, platelets were always counted in the plasma. Before analysis, plasma samples (500 $\mu \mathrm{l})$ were deproteinized with $100 \mu \mathrm{l}$ of $24 \%(\mathrm{w} / \mathrm{v})$ 5 -sulphosalicylic acid, containing $2 \mathrm{mmol} / 1$ norvaline and homoserine. After immediate vortexmixing and standing for $15 \mathrm{~min}$ at $4^{\circ} \mathrm{C}$, the precipitate was spun down (15 min at 18400 $\left.g_{\text {max }}\right)$. Next, $400 \mu \mathrm{l}$ of the supernatant was transferred to a $0.8-\mathrm{ml}$ polypropylene vial and was mixed with $100 \mu \mathrm{l}$ of $0.3 \mathrm{M} \mathrm{LiOH}$ in order to adjust the $\mathrm{pH}$ to 2.5 .

\subsection{Derivatization procedure}

An automated precolumn derivatization procedure was employed using the Gilson Autosampling injector, equipped with a coldblock $\left(3^{\circ} \mathrm{C}\right)$ and with rack code 31 . The following program was created: first, aspirate a 5- $\mu$ l air bubble into the transferring tube, then aspirate $20 \mu \mathrm{l}$ of the OPA-reagent, followed by $5 \mu \mathrm{l}$ air and $5 \mu 1$ of sample or standard. The total volume was dispensed into an empty vial and mixed by aspirating and dispensing twice. After waiting 6 $s$, the total volume was aspirated into the transferring tube and the sample loading line was flushed with $15 \mu \mathrm{l}$ of this mixture. Next, the filling loop was loaded with $2 \mu \mathrm{l}$, and 3 s later the valve was switched to inject position. After each injection cycle the sample loading line was flushed with $300 \mu$ l of $10 \%$ methanol. The volumetric precision obtained with this partial loop filling procedure was $0.5-1.5 \%$.

\subsection{Chromatographic conditions}

A ternary solvent system was used. The gradient program for the Spherisorb ODS 2 cartridge column $(5 \mu \mathrm{m})$ is shown in Table 1 . The flow-rate was maintained at $1.0 \mathrm{ml} / \mathrm{min}$ throughout and the column temperature was $25^{\circ} \mathrm{C}$. Typical back pressures ranged from 17-19 $\mathrm{MPa}$ with solvents $\mathrm{A}$ and $\mathrm{B}$ to $20-22 \mathrm{MPa}$ with solvent $\mathrm{C}$. Total run time (injection to injection) was $49 \mathrm{~min}$. For the other columns tested, the flow-rate varied between 0.5 and $2.5 \mathrm{ml} / \mathrm{min}$ (depending on the diameter and particle size), the temperature ranged between 25 and $35^{\circ} \mathrm{C}$ and the gradient program was also slightly different. Amino acid derivatives were identified by their retention times relative to the reference peaks homoserine and norvaline. Quantitation was done by measuring peak heights relative to the internal standard norvaline.

\subsection{Assay performance}

Quality of the analytical data was assessed by assaying plasma samples that had been sup-

Table 1

Gradient conditions

\begin{tabular}{ccc}
\hline $\begin{array}{l}\text { Time } \\
(\min )\end{array}$ & $\begin{array}{l}\mathrm{B} \\
(\%)\end{array}$ & $\begin{array}{l}\mathrm{C} \\
(\%)\end{array}$ \\
\hline 0 & 0 & 0 \\
13.0 & 0 & 0 \\
13.8 & 0 & 1.8 \\
14.0 & 97.8 & 2.2 \\
24.0 & 90.0 & 10.0 \\
26.0 & 0 & 13.3 \\
28.0 & 0 & 20 \\
40.0 & 0 & 57 \\
41.0 & 0 & 59 \\
44.0 & 0 & 82 \\
45.8 & 0 & 100 \\
46.0 & 0 & 0 \\
\hline
\end{tabular}


plemented with appropriate amino acids. The HPLC results were also compared with those acquired using an LKB 4400 Amino Acid Analyzer with fluorescence detection [14]. Moreover, plasma samples were assayed which had been analyzed by other laboratories in accordance with the terms of the European Quality Assurance Program.

\section{Results and discussion}

We modified the solvents and gradient used by Turnell and Cooper [1], to separate over 40 amino acids in a plasma-based standard. The addition of tetrahydrofuran instead of dimethylsulfoxide to the buffered solvents $A$ and $B$, resulted in a better resolution between histidine and glutamine. Moreover, this resolution remained satisfactory for a much longer time. Furthermore, we made use of an oven with built-in Peltier cooling, resulting in a very stable column temperature of $25^{\circ} \mathrm{C}$ and consequently stable retention times. This enabled us to quantify the amino acid derivatives by measuring peak heights, which is more accurate in case of unresolved peaks. With the cooled sample-compartment, 48 analyses can be done automatically and unattended.

Of the various columns tested, the best resolution was achieved with both Spherisorb ODS 2 cartridge columns ( 3 and $5 \mu \mathrm{m}, 125 \times 3 \mathrm{~mm}$ I.D.) from Phase Separations, the Spherisorb ODS 2 column $(5 \mu \mathrm{m}, 150 \times 4.6 \mathrm{~mm}$ I.D.) from Shandon and the Supelcosil LC-18 column (5 $\mu \mathrm{m}, 150 \times 4 \mathrm{~mm}$ I.D.) from Supelco. The problem with the other columns was that the separation between valine and methionine was unsatisfactory, except for the Hypersil-ODS column from Hewlett-Packard. However, the latter column as well as the Inertsil and Zorbax columns showed inferior resolution of the cluster of amino acids between glycine and hypotaurine. The Spherisorb ODS 2 columns from Shandon with the smaller diameters also had some problems with the resolution between threonine and 1-methylhistidine and allo-L-cystathionine and phenylalanine. Moreover, the resolution of his- tidine-glutamine became worse after some 100 injections. The Hypersil Amino Acid column showed, next to the bad valine-methionine resolution, unsatisfactory separation between 1methylhistidine and arginine.

We chose the Spherisorb ODS 2 cartridge column with $5-\mu \mathrm{m}$ particles as the standard column, because the other columns did have some drawbacks. The Spherisorb ODS 2 cartridge column packed with $3-\mu \mathrm{m}$ particles exhibited extremely high backpressures, $35 \mathrm{MPa}$ with $0.85 \mathrm{ml} / \mathrm{min}$ solvent $\mathrm{C}$ at $25^{\circ} \mathrm{C}$. For the Spherisorb ODS 2 column from Shandon, a rather high flow-rate $(2.5 \mathrm{ml} / \mathrm{min})$ was necessary and the separations between histidine and glutamine and between arginine and $\beta$-alanine became worse after some 200 injections. With the Supelcosil LC-18 column, the separations between citrulline and 3-methylhistidine and between valine and methionine were unsatisfactory. Moreover, the flow-rate for this column $(1.9 \mathrm{ml} / \mathrm{min})$ was also higher than for the Spherisorb ODS 2 cartridge column $(1.0 \mathrm{ml} /$ $\min$ ).

An amino acid chromatogram of the standard containing 40 amino acids (representative of a physiological sample) is shown in Fig. 1. It can be seen that, except for carnosine and anserine, all amino acids show excellent separation. It is also possible with this method to detect argininosuccinic acid (retention time $4.5 \mathrm{~min}$ ), $\beta$-aminobutyric acid (retention time $34.0 \mathrm{~min}$ and eluting between $\tau$-aminobutyric acid and tyrosine) and diaminobutyric acid (retention time $45.7 \mathrm{~min}$ and eluting between hydroxylysine and ornithine). However, cysteine, proline and hydroxyproline could not be detected with OPA derivatization. We did not pretreat our samples with iodoacetic acid in order to detect cysteine, because this may lead to incorrect values of the more important amino acids tyrosine and histidine [15]. On the other hand, we added Brij 35 to the derivatizing reagent to increase the fluorescence response of lysine and hydroxylysine [16]. The problem of the instability of the OPA-reagent [6] was solved by using multiple amber crimp top vials with a silicone rubber, PTFE-coated cap. Each vial was used for eight consecutive samples. 


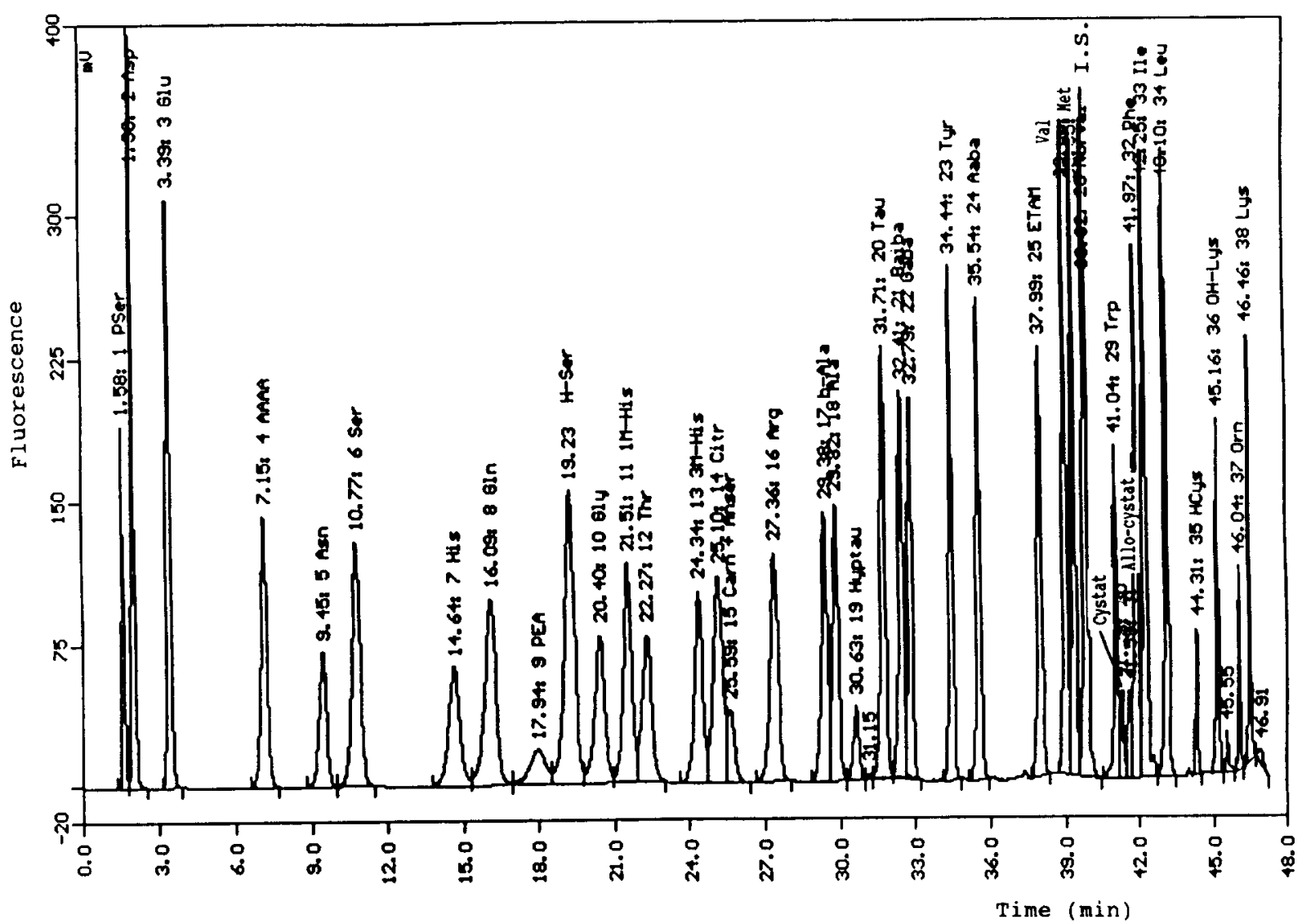

Fig. 1. Elution profile of an OPA-derivatized physiological amino acid standard (approx. 50 pmol per amino acid). Column: Spherisorb ODS $2(125 \times 3 \mathrm{~mm}$ I.D.); flow-rate $1.0 \mathrm{ml} / \mathrm{min}$. For eluents and gradient, see Section 2 and Table 1 , respectively. Non-standard abbreviations used: Pser $=$ phosphoserine; AAAA $=\alpha$-aminoadipic acid; PEA $=$ phosphoethanolamine; $\mathrm{H}-\mathrm{Ser}=$ homoserine: Carn = carnosine; Anser $=$ anserine; $b$-Ala $=\beta$-alanine; Hyptau $=$ hypotaurine; Baiba $=\beta$-aminoisobutyric acid; $\mathrm{Gaba}=\tau$-aminobutyric acid; Aaba $=\alpha$-aminobutyric acid; ETAM $=$ ethanolamine; I.S. = internal standard (= norvaline); cystat $=$ cystathionine $;$ allo-cystat $=$ allo-cystathionine $;$ HCys $=$ homocystine $;$ OH-Lys $=$ hydroxylysine .

It has to be noticed that there is some batchto-batch variation. This sometimes leads to a bad separation between citrulline and carnosine/anserine and between allo-L-cystathionine and phenylalanine. However, since we make use of peak height determination for the quantitation, this is not a serious problem. In the rare cases where plasma contains carnosine or anserine, we are able to notice this by comparing the peak widths of citrulline in the standard and the sample. A slight adjustment of the gradient condition by changing the time of the start of solvent $\mathrm{B}$ a few minutes or changing the $\mathrm{pH}$ of this solvent by 0.03 units, results in a satisfactory resolution between citrulline and carnosine/anserine. If the methylhistidines are not fully resolved, lowering of the $\mathrm{pH}$ of solvent $\mathrm{B}$ by $0.02-0.04$ units or starting the gradient with this solvent a few minutes earlier, will improve their resolution. The resolution between allo-Lcystathionine and phenylalanine can be improved by increasing the percentages of acetonitrile and methanol in solvent $\mathrm{C}$ by 2 and $1 \%$, respectively.

The coefficient of variation (C.V.) for the retention times was below $0.5 \%$ within one batch of analyses. The within-run precision was determined both for the physiological standard and 
a pooled plasma. The C.V. was for all amino acids below $4 \%$. Between-run precision was estimated by analyzing 3 aliquots of human plasma on 10 different days. The C.V. was for most amino acids below 4\%, except for 1methylhistidine $(13.5 \%)$, citrulline $(4.4 \%), 3-$ methylhistidine $(27 \%)$, taurine $(4.4 \%), \beta$ aminoisobutyric acid $(60 \%), \alpha$-aminobutyric acid $(5.6 \%)$ and methionine $(4.6 \%)$.

Under the conditions described, the peak heights are linear with concentration up to 1200 $\mu \mathrm{mol} / \mathrm{l}$ for each amino acid. Concentrations of amino acids down to $0.5 \mu \mathrm{mol} / 1$ in plasma can be measured accurately with our method. This means that trace amino acids like $\alpha$-aminoadipic acid, phosphoethanolamine, 1- and 3-methylhistidine, $\beta$-alanine, hypotaurine, $\beta$-aminoisobutyric acid, $\tau$-aminobutyric acid and cystathionine can also be determined. The limit of detection depends on the amino acid in question, because of different fluorescence responses and differing peak shapes of the derivatives. Typical values were $53 \mathrm{fmol}$ for aspartic acid and $167 \mathrm{fmol}$ for serine.

In Fig. 2, a typical chromatogram of plasma is given, showing that 28 physiological amino acids could be satisfactory separated, identified and quantified. The mean amino acid concentrations in plasma from 44 healthy subjects are presented in Table 2. The results are in very good agreement with values obtained by other investigators $[4,6-8,13,17]$. A comparison between our HPLC method and the conventional amino acid analyzer for the same samples is included in Table 2. The HPLC results compared favourably with those obtained with the amino acid analyzer.

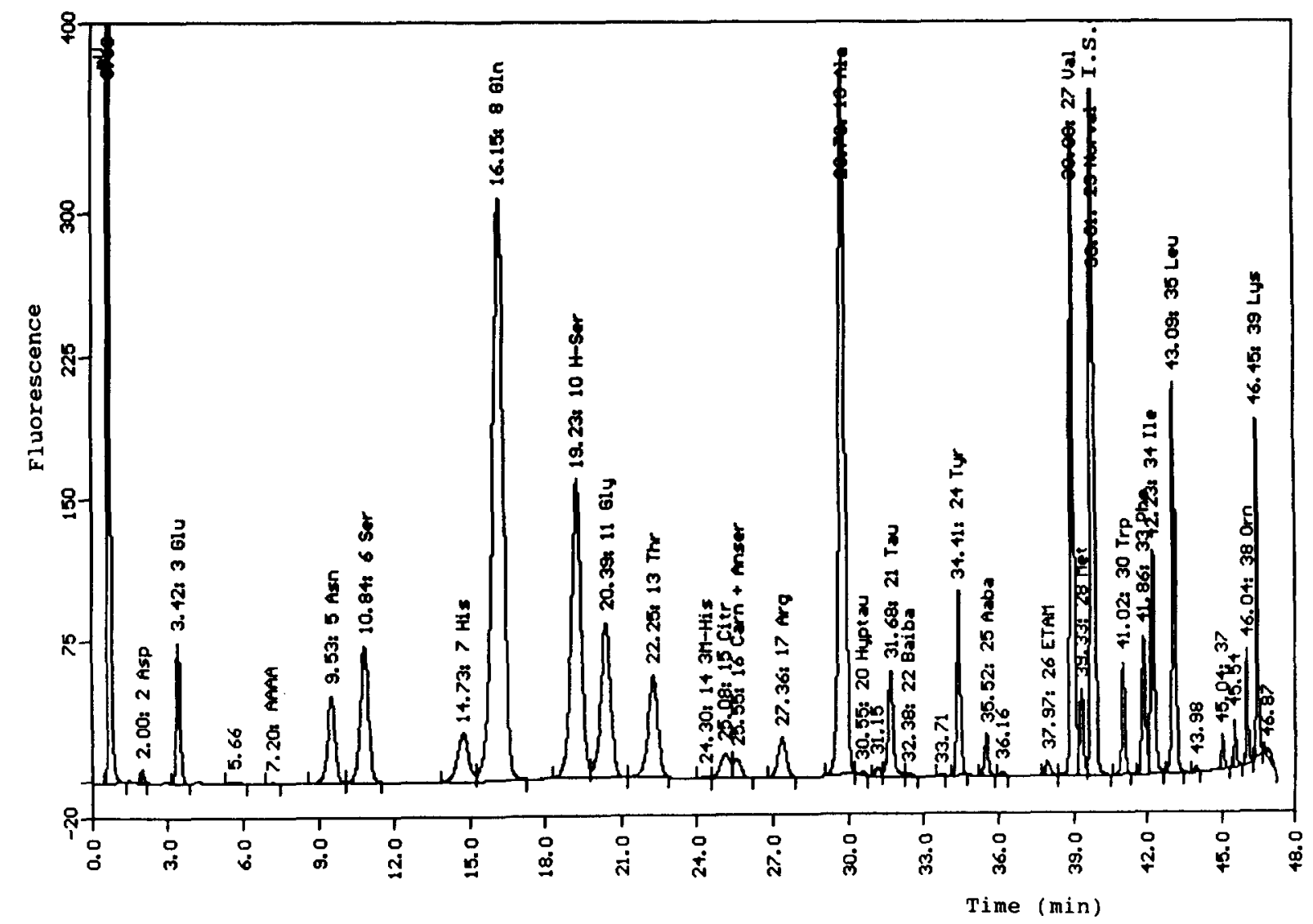

Fig. 2. Chromatogram of human plasma amino acids from a healthy volunteer under identical experimental conditions as in Fig. 1. For abbreviations see Fig. 1. 
Table 2

Amino acid concentrations in plasma from 44 healthy volunteers as determined by HPLC and ion-exchange chromatography (values are given in $\mu \mathrm{mol} / \mathrm{l}$ as mean \pm S.D.)

\begin{tabular}{|c|c|c|}
\hline Amino acid & HPLC & Ion-exchange ${ }^{a}$ \\
\hline Aspartic acid & $4.5 \pm 2.5$ & $5.4 \pm 3.0$ \\
\hline Glutamic acid & $52.0 \pm 18.0$ & $36.0 \pm 12.0$ \\
\hline$\alpha$-Aminoadipic acid & $0.3 \pm 0.4$ & N.D. ${ }^{h}$ \\
\hline Asparagine & $54.0 \pm 8.0$ & $58.0 \pm 14.0$ \\
\hline Serine & $104.0 \pm 16.0$ & $99.0 \pm 20.0$ \\
\hline Histidine & $82.0 \pm 11.0$ & $92.0 \pm 13.0$ \\
\hline Glutamine & $570.0 \pm 69.0$ & $576.0 \pm 74.0$ \\
\hline Phosphoethanolamine & $2.0 \pm 2.3$ & $1.0 \pm 1.4$ \\
\hline Glycine & $196.0 \pm 42.0$ & $202.0 \pm 48.0$ \\
\hline Threonine & $137.0 \pm 24.0$ & $133.0 \pm 33.0$ \\
\hline 1-Methylhistidine & $5.6 \pm 4.2$ & $8.2 \pm 8.6$ \\
\hline Citrulline & $36.0 \pm 7.4$ & $31.0 \pm 6.5$ \\
\hline 3-Methylhistidine & $10.3 \pm 4.9$ & $7.0 \pm 5.1$ \\
\hline Arginine & $81.0 \pm 15.0$ & $79.0 \pm 24.0$ \\
\hline$\beta$-Alanine & $3.2 \pm 2.3$ & $4.1 \pm 1.6$ \\
\hline Alanine & $379.0 \pm 75.0$ & $399.0 \pm 71.0$ \\
\hline Taurine & $46.0 \pm 7.9$ & $50.0 \pm 11.0$ \\
\hline$\beta$-Aminoisobutyric acid & $4.2 \pm 2.5$ & $1.6 \pm 1.8$ \\
\hline Tyrosine & $67.0 \pm 11.0$ & $63.0 \pm 19.0$ \\
\hline$\alpha$-Aminobutyric acid & $24.0 \pm 6.4$ & $22.0 \pm 6.2$ \\
\hline Ethanolamine & $7.0 \pm 1.0$ & $11.0 \pm 1.8$ \\
\hline Valine & $285.0 \pm 48.0$ & $251.0 \pm 56.0$ \\
\hline Methionine & $29.0 \pm 4.6$ & $27.0 \pm 6.9$ \\
\hline Tryptophan & $57.4 \pm 7.2$ & $55.2 \pm 9.5$ \\
\hline Phenylalanine & $61.0 \pm 7.8$ & $55.0 \pm 6.9$ \\
\hline Isoleucine & $82.0 \pm 16.0$ & $71.0 \pm 14.0$ \\
\hline Leucine & $142.0 \pm 23.0$ & $135.0 \pm 24.0$ \\
\hline Ornithine & $73.0 \pm 15.0$ & $72.0 \pm 19.0$ \\
\hline Lysine & $180.0 \pm 27.0$ & $171.0 \pm 26.0$ \\
\hline
\end{tabular}

"Ion-exchange chromatography was done using an LKB 4400 Amino Acid Analyzer and post-column derivatization with OPA $[14]$.

"N.D. $=$ not detected.

The actual correlation between the two methods was highly significant $(r=0.997, p<0.001)$.

The quality of the analytical data was assessed by assaying plasma samples that had been supplemented with known amounts of amino acids and by assaying plasma samples in which amino acid concentrations had been determined by several laboratories. The recovery of each amino acid ranged between 96 and $105 \%$, except for tryptophan $(87 \%)$. These values are in accordance with literature values $[7,9,13]$. The concentrations of amino acids given in Table 2 have all been corrected for recovery. However, one may argue that correction for recovery of amino acids-except for tryptophan-has any value, since concentrations in the physiological amino acid standard solutions supplied by Sigma are $\pm 4 \%$.

The results of the quality study are given in Table 3. It can be seen that the rare amino acids $\alpha$-aminoadipic acid, $\beta$-alanine, $\beta$-aminoisobutyric acid, ethanolamine and cystathionine showed the most deviating values. Nevertheless, even when all 30 amino acids were taken into account, our mean values were generally in very good agreement with the mean and median values of other laboratories. Correlation coefficients were $r=0.993$ and $r=0.996$, respectively. 
Table 3

HPLC analyses of amino acids in spiked plasma: comparison with other laboratories (results are given in $\mu$ mol/l and have not been corrected for recovery)

\begin{tabular}{|c|c|c|c|c|c|c|c|}
\hline \multirow[t]{2}{*}{ Amino acid } & \multicolumn{3}{|l|}{ HPLC } & \multicolumn{4}{|c|}{ Other laboratories $^{A}$} \\
\hline & Mean & S.D. & $n$ & Mean & Median & S.D. & $n$ \\
\hline Aspartic acid & 24.3 & 0.9 & 11 & 24.8 & 25.0 & 7.7 & 511 \\
\hline Glutamic acid & 220.0 & 0.5 & 11 & 233.0 & 227.0 & 62.1 & 521 \\
\hline$\alpha$-Aminoadipic acid & 3.3 & 0.5 & 8 & 6.3 & 4.0 & 5.4 & 57 \\
\hline Asparagine & 101.0 & 1.6 & 11 & 111.0 & 100.0 & 53.0 & 460 \\
\hline Serine & 66.2 & 1.4 & 11 & 63.9 & 63.0 & 11.9 & 526 \\
\hline Histidine & 47.2 & 0.9 & 11 & 45.6 & 45.0 & 9.6 & 503 \\
\hline Glutamine & 334.0 & 11.8 & 11 & 322.0 & 308.0 & 112.0 & 505 \\
\hline Glycine & 234.0 & 6.2 & 11 & 229.0 & 230.0 & 33.4 & 526 \\
\hline Threonine & 249.0 & 2.2 & 11 & 235.0 & 239.0 & 34.7 & 536 \\
\hline 1-Methylhistidine & 5.8 & 0.8 & 6 & 5.0 & 5.0 & 3.0 & 98 \\
\hline Citrulline & 33.3 & 1.2 & 3 & 38.7 & 37.0 & 12.3 & 498 \\
\hline 3-Methylhistidine & 23.2 & - & 1 & 21.0 & 20.0 & 5.2 & 388 \\
\hline Arginine & 120.0 & 2.2 & 11 & 119.0 & 117.0 & 24.7 & 521 \\
\hline$\beta$-Alanine & 2.0 & 0.0 & 2 & 5.0 & 4.0 & 3.2 & 26 \\
\hline Alanine & 565.0 & 6.9 & 11 & 551.0 & 565.0 & 82.5 & 538 \\
\hline Taurine & 46.5 & 2.0 & 11 & 49.1 & 48.0 & 8.4 & 481 \\
\hline$\beta$-Aminoisobutyric acid & 2.9 & 2.4 & 7 & 89.7 & 61.5 & 93.4 & 22 \\
\hline Tyrosine & 37.4 & 0.8 & 11 & 38.8 & 36.0 & 11.2 & 531 \\
\hline$\alpha$-Aminobutyric acid & 7.4 & 0.5 & 11 & 6.4 & 5.5 & 4.4 & 236 \\
\hline Ethanolamine & 10.7 & 0.6 & 3 & 40.1 & 29.0 & 33.1 & 34 \\
\hline Valine & 261.0 & 1.8 & 11 & 240.0 & 245.0 & 40.6 & 533 \\
\hline Methionine & 44.6 & 1.4 & 11 & 52.4 & 52.0 & 12.3 & 536 \\
\hline Tryptophan & 24.6 & 0.5 & 11 & 25.4 & 25.0 & 8.6 & 227 \\
\hline Cystathionine & 11.8 & 0.5 & 4 & 27.3 & 12.0 & 34.5 & 171 \\
\hline Phenylalanine & 427.0 & 7.4 & 11 & 404.0 & 405.0 & 59.2 & 538 \\
\hline Isoleucine & 64.7 & 0.5 & 7 & 63.7 & 61.0 & 17.3 & 527 \\
\hline Leucine & 226.0 & 3.1 & 11 & 220.0 & 224.0 & 39.3 & 544 \\
\hline Homocystine & 45.0 & 2.8 & 3 & 43.5 & 45.0 & 24.6 & 172 \\
\hline Ornithine & 52.4 & 1.2 & 11 & 53.0 & 51.0 & 14.7 & 517 \\
\hline Lysine & 445.0 & 11.3 & 11 & 427.0 & 428.0 & 68.1 & 531 \\
\hline
\end{tabular}

${ }^{a} 89$ Laboratories participating in the European Quality Assurance Program.

When $\beta$-aminoisobutyric acid, the amino acid with the highest C.V., was excluded, the correlation coefficient reached the high value of $r=$ 0.999 . So, it can be concluded that the results obtained with our HPLC method are in excellent agreement with those of other laboratories.

Apart from the fact that precipitation of plasma proteins with SSA does not result in good recovery of tryptophan, the concentrations of amino acids found with our method are in very good agreement with the literature values [4,6$8,13,17]$. Another deproteinization method used by several investigators, precipitation with acetonitrile in combination with the OPA method, does not always result in reliable values [1$3,5,7]$. This is probably due to proteolysis and/or incomplete extraction [5] or to chemical instability [7]. However, it has to be noticed that in these studies OPA was used as the derivatization reagent. When the phenylisothiocyanate derivatization method was used in combination with acetonitrile deproteinization, results obtained were more accurate, although C.V. values for some amino acids were still rather high [18]. On 
the other hand, deviating physiological values may also result from improper identification of amino acids $[2,5,18]$.

As many as 250 successive runs could be performed with the Spherisorb cartridge column without any deterioration of resolution. When the separation efficiency decreased, we flushed the column with acetonitrile or dichloromethane to restore column performance. When this decrease was accompanied by an increase in the back-pressure, we also changed the guard column. The advantage of using this cartridge system is the low cost, and the reusable endfittings make switching of columns both rapid and simple.

Since we can separate over 40 amino acids, there is a very small chance that amino acids are overestimated, wrongly identified or not identified at all. Therefore, our method can also be applied to the determination of amino acids in serum, urine, cerebrospinal fluid and tissues. An example of an amino acid analysis from a urine sample is shown in Fig. 3.

In the literature, only two groups report on an assay for amino acids in human plasma capable of separating $40[10]$ or more [2] amino acids. However, the first group [10] did not report normal values and their analysis takes $67 \mathrm{~min}$. Although the second group [2] was able to separate 48 amino acids within $50 \mathrm{~min}$, their normal values for most amino acids were far below the true physiological values. The three

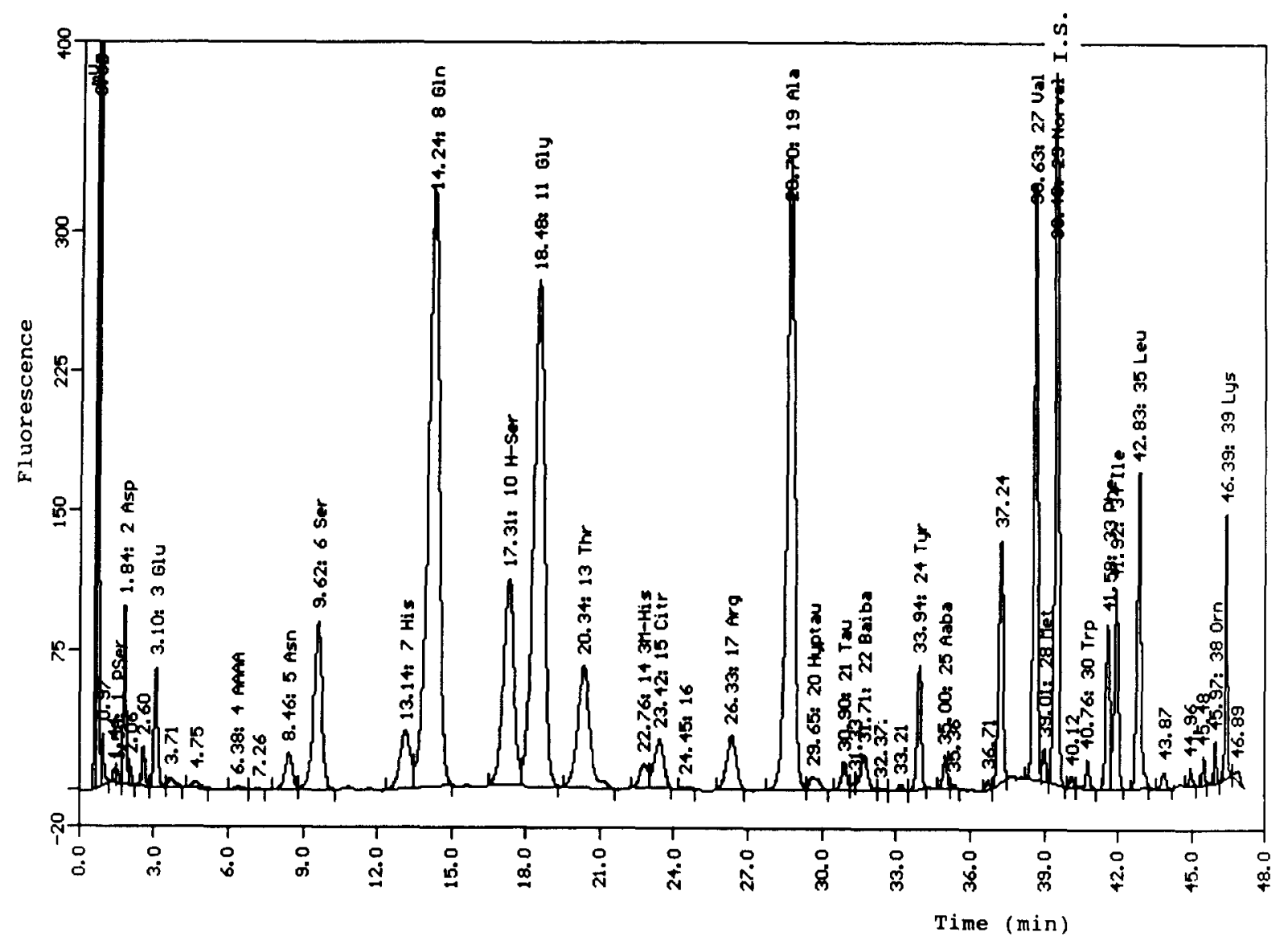

Fig. 3. Elution profile of OPA-derivatized amino acids from adult urine. Chromatographic conditions and non-standard abbreviations as in Fig. 1. 
groups who report on very short analysis times $[5,1213]$ are not able to separate physiological trace amino acids like $\beta$-aminoisobutyric acid, $\beta$-alanine and cystathionine. Besides, one of these groups does not even resolve tryptophan and ornithine [5].

\section{Acknowledgements}

We thank Dr. Ph. Parvy and Dr. P. Kamoun for their permission of using the amino acid data obtained in accordance with the terms of the European Quality Assurance Program. We also thank Dr. J.G.M. Huijmans for providing us with the plasma samples used for the quality study.

\section{References}

[1] D.C. Turnell and J.D.H. Cooper, Clin. Chem., 28 (1982) 527

[2] B.N. Jones and J.P. Gilligan, J. Chromatogr., 266 (1983) 471.

[3] M.O. Fleury and D.V. Ashley, Anal. Biochem., 133 (1983) 330
[4] H.G. Godel, T.A. Graser, P. Foldi, P. Pfaender and P. Fürst, J. Chromatogr., 297 (1984) 49.

[5] T.A. Graser, H.G. Godel, S. Albers, P. Foldi and P. Fürst, Anal. Biochem., 151 (1985) 142.

[6] H.M.H. van Eijk, M.A.H. van der Heijden, C.L.H. van Berlo and P.B. Soeters, Clin. Chem., 34 (1988) 2510.

[7] G.A. Qureshi and A.R. Qureshi, J. Chromatogr., 491 (1989) 281.

[8] P. Fürst, L. Pollack, T.A. Graser, H.G. Godel and P. Stehle, J. Chromatogr., 499 (1990) 557.

[9] A.M. Uhe, G.R. Collier, E.A. McLennan, D.J. Tucker and K. O'Dea, J. Chromatogr., 564 (1991) 81.

[10] J. Schmidt and C.J. McClain, J. Chromatogr., 568 (1991) 207.

[11] G. Georgi, C. Pietsch and G. Sawatzki, J. Chromatogr., 613 (1993) 35.

[12] H.M.H. van Eijk, D.R. Rooyakkers and N.E.P. Deutz, J. Chromatogr., 620 (1993) 143.

[13] T. Teerlink, P.A. van Leeuwen and A. Houdijk, Clin. Chem., 40 (1994) 245.

[14] J. Bruinvels and L. Pepplinkhuizen, Psychiatr. Res., 18 (1984) 307.

[15] J.D.H. Cooper and D.C. Turnell, J. Chromatogr., 227 (1982) 158.

[16] J.R. Benson and P.E. Hare, Proc. Natl. Acad. Sci. USA, 72 (1975) 619.

[17] I. Fermo, E. De Vecchi, L. Diomede and R. Paroni, J. Chromatogr., 534 (1990) 23.

[18] G. Sarwar and H.G. Botting, J. Chromatogr., 615 (1993) 1. 